General Mathematics Vol. 28, No. 2 (2020), 103-104

DOI: $10.2478 / \mathrm{gm}-2020-0020$

sciendo

\title{
Book Review: Approximation with Positive Linear Operators and Linear Combinations By: Vijay Gupta, Gancho Tachev Series: Developments in Mathematics, Volume 50, Springer, Cham, 2017. 1
}

\author{
Ana Maria Acu
}

\section{Book Review}

The book is devoted to a detailed study on approximation by positive linear operators and it is addressed to students in mathematics, but also to any researchers and $\mathrm{PhD}$ students interested in the field of approximation theory and its applications. The first two chapters deal with the moments and the central moments of some positive linear operators. Since the moments play an important role in approximation theory by positive linear operator, this book can be used successfully in the research work.

The known strong converse inequalities of type A in the terminology of DitzianIvanov for linear combinations of Bernstein and Bernstein-Kantorovich operators are presented. Some open problems concerning the approximation by linear combinations of positive linear operators are outlined.

In recent years, there is an increasing interest to give quantitative estimates for positive linear operators in approximating the functions. The Voronovskaja-type theorem is one of the most important result which describes the rate of pointwise convergence. In this book some of the results appeared in the recent years on such problems are very well described.

The book is very well written, structured and organized. All the notions and results are clearly presented. The book is highly recommended as well as for self-study by researchers needing a quick access to some top research tools in approximation theory.

\footnotetext{
${ }^{1}$ Received 20 June, 2020

Accepted for publication (in revised form) 6 September, 2020
} 
Ana Maria Acu

Lucian Blaga University of Sibiu

Department of Mathematics and Informatics

Str. Dr. I. Ratiu, No.5-7, RO-550012 Sibiu, Romania

e-mail: anamaria.acu@ulbsibiu.ro 